\title{
The H-Mode of ASDEX
}

\section{A favourable operational regime of tokamak plasmas at near-ignition conditions}

\section{F. Wagner, Garching}

(Max-Planck-Institut für Plasmasphysik)

The conditions for a magnetically confined plasma to ignite are a plasma temperature above 100 Million degree (10 $\mathrm{keV}$ ) and a product of density $n_{\mathrm{e}}$ and energy confinement time $\tau_{E}$ in excess of $2 \times 10^{20} \mathrm{~m}^{-3} \mathrm{~s}$. (Technical requirements such as low plasma contamination by impurity ions have additionally to be fulfilled.) Under steady state conditions, the energy confinement time determines the energy content of the discharge at given heating power or describes its decay when the heating power is switched-off. On the large tokamak at Princeton, the PLT, an ion temperature of $7.5 \mathrm{keV}$ was achieved in 1979 with natural injection (NI) auxiliary heating ${ }^{1}$ ) and on Alcator $\mathrm{C}$, another tokamak device in the USA, using resistive heating by the plasma current (the same process which heats a normal conductor), the less critical $n_{e} \tau_{E}$ breakeven limit was reached in 1984, albeit with a low temperature of $1.5 \mathrm{keV}$. Thus the road to an ignited tokamak plasma seemed to be clear.

However there turned out to be an unexpected road block: the good confinement properties of resistively heated plasmas could not be maintained at high plasma temperatures. With increasing auxiliary heating power, the plasma confinement was found to degrade severely and ignition conditions were in effect not approached. This degradation in confinement was a world-wide observed phenomenon which was found to be independent of the heating technique and which seemed to threaten the ultimate goal of the fusion programme.

On ASDEX, the AxiSymmetric Divertor tokamak EXperiment at Garching, the same problem was encountered: $\tau_{\mathrm{E}}$ decreased from $70 \mathrm{~ms}$, established in a resistively heated discharge with a typical Ohmic input of $0.4 \mathrm{MW}$, to $20 \mathrm{~ms}$ during a NI pulse of $3 \mathrm{MW}$. The particle confinement time was also observed to decrease. However, on ASDEX a solution of this problem was found by surrounding the plasma with a thermal insulation layer. This solution to the heat leak problem is in itself not unique every house owner applies it in an effort to increase the room temperatures (improve the energy confinement time) without having to uppgrade the furnace.

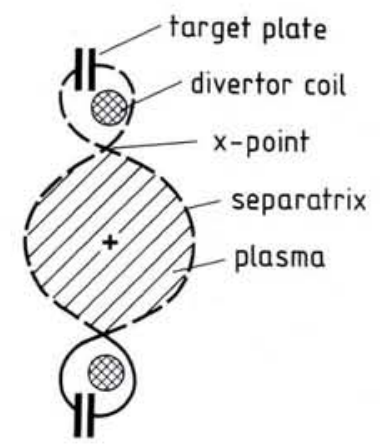

Fig. 1 - Poloidal cross-section of the dou ble-null divertor configuration of ASDEX.

We should not feel inclined to report on it if the thermal resistivity of the insulation layer surrounding the ASDEX plasma were not to surpass that of a product such as Styrofoam by three orders of magnitude, and if degraded confinement as a limitation of the present experiments were not thereby mitigated.

\section{Design Features of ASDEX}

The toroidal plasma in ASDEX has major and minor radii of $1.65 \mathrm{~m}$ and 0.4 $\mathrm{m}$; maximum plasma current is $0.5 \mathrm{MA}$; stabilization is provided by a toroidal magnetic field of up to $2.8 \mathrm{~T}$. The NI system of ASDEX delivers a beam of hydrogen atoms with $40 \mathrm{keV}$ energy and with a maximum power of $4.4 \mathrm{MW}$. The main research goal of ASDEX is to demonstrate the efficiency of the magnetic di- vertor concept in providing ultraclean hydrogen discharges by minimizing the interaction of the hot plasma with the surrounding vessel wall.

In most tokamak designs, as in JET for example ${ }^{2}$ ), the plasma wall contact is reduced by the inclusion of a poloidal diaphragm, the so-called limiter, whose opening defines the minor plasma cross-section. The reduced wall erosion is, however, at the expense of intense limiter interaction. In the case of ASDEX, the material limiter has been replaced by a magnetic surface, the separatrix, as shown in Fig. 1. The separatrix is formed by the superposition of the magnetic fields of the plasma current and currents running in the toroidal divertor coils. For unidirectional currents the magnetic fields cancel at the X-points. There the magnetic field lines do not helically wind around the torus but point only in the direction of the toroidal magnetic field. Approaching the $X$-point, the field line inclination changes quickly and the path length for a poloidal circulation increases.

The separatrix defines the plasma surface by separating the internal magnetic field structure - characterized by nested toroidal magnetic surfaces - from the open field lines at the outside (scrape-off layer) which are bent away from the plasma into the divertor chamber where they intersect target plates. Inside the separatrix, the plasma pres-
Fig. 2 - Comparison of the energy content $W(a, b)$ and particle content $N(c, d)$ of L-type $(a, c)$ and H-type $(b, d)$ plasmas. The Nl-pulse is indicated by the hatched interval.
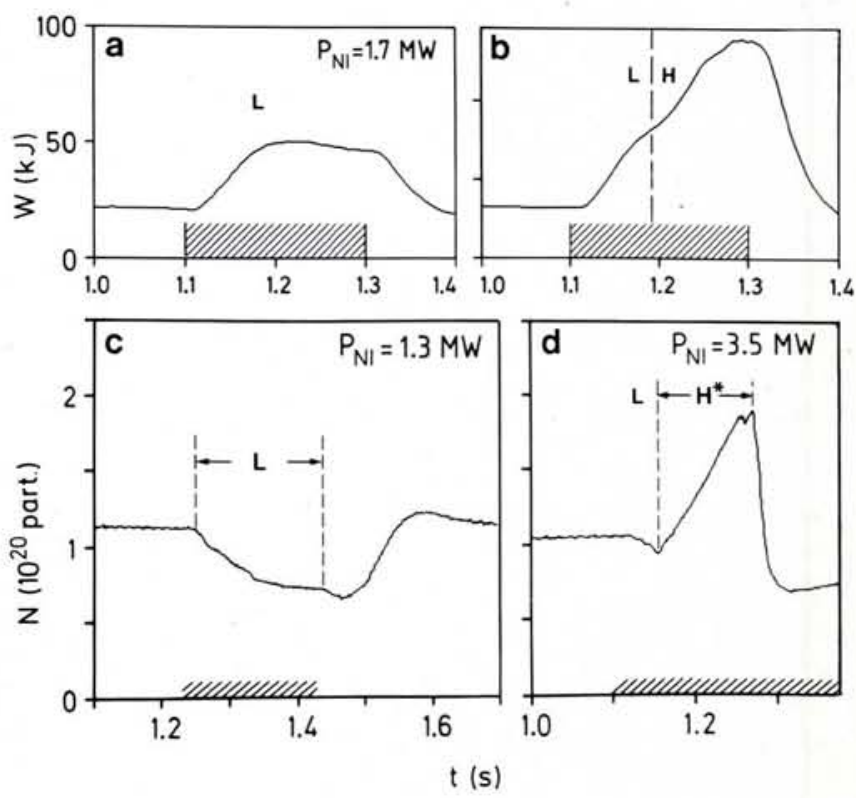
sure is high and determined by the low transport properties across flux surfaces; in the scrape-off layer, the plasma is quickly lost to the target plates due to the rapid transport along field lines. Thus a sharp plasma boundary develops at the separatrix. Energy and particle fluxes out of the main plasma flow along the scrape-off layer into the divertor chamber. ASDEX has both demonstrated the possibility of the divertor concept and produced plasmas with a low degree of contamination. This has been described in Ref. 3.

\section{Confinement Properties During NI}

Injecting a neutral beam into ASDEX plasmas gives rise to two confinement regimes. Their important features are summarized in Fig. 2 which shows the development of the energy content $W$ and the particle content $N$ during an injection pulse. The discharges (2a) and (b) have identical plasma and beam parameter settings. The heating power is increased from the Ohmic level by a factor of 4.5. In case (a) $W$ increases by a factor of 2.5 only indicating degraded confinement, whereas in case (b), $W$ increases in proportion to the heating power. Case (a) is termed L-(for low), type (b) $\mathrm{H}$-(for high) discharge ${ }^{4}$ ) because of their respective low and high confinement properties. Although the crucial element which gives rise to the $\mathrm{L}$ - or $\mathrm{H}$ mode is not yet precisely known (see next section), the transition from $L$ to $H$ is sharp and the plasma behaviour in the two modes is quite distinct. Figures $2 c$ and $2 \mathrm{~d}$ demonstrate the impact of changes in the particle confinement on the plasma particle content. In the L-discharge, the plasma rapidly loses nearly half of its particle content; in the $\mathrm{H}$ phase, $N$ increases steeply. An interesting feature of an $\mathrm{H}$-discharge is that it always develops out of a preceding short L-phase as indicated in Figs. $2 b$ and $d$. The transition into the $\mathrm{H}$-phase is distinct, pointing toward the existence of precise transition conditions. The important aspect of the $\mathrm{H}$-phase is that the enhanced electron heat transport of the Lphase is suddenly reduced giving rise to good confinement properties. Although the global confinement times in the $\mathrm{H}$ phase are comparable to the Ohmic values, the plasma does not simply readopt the Ohmic transport characteristics. In an H-plasma, as in all high power auxiliary heated tokamak plasmas, power deposition and current density profiles are different. Moreover, also in $\mathrm{H}$-plasmas the leading scaling parameter of $\tau_{E}$ is the current, and not the plasma density as in Ohmic discharges.

\section{Research in X-ray Diffraction using Synchrotron Radiation}

\section{The Job}

A vacancy exists for a senior experimental research scientist (male/female) to develop. operate and exploit $x$-ray diffraction facilities using the synchrotron radiation source. We already have a two-circle diffractrometer, and we are buying a four-circle instrument. One of the initial tasks will be to develop their use for $2 \mathrm{D}$ surface studies.

The duties will involve coordination with university experimental teams, the direction and supervision of Laboratory staff and responsibility for an operating budget. In addition the postholder would be required to take active part in Laboratory management.

\section{The Person}

We need someone with extensive experimental and theoretical experience in $\mathrm{x}$-ray diffraction applied to the physical sciences and preferably with experience in the use of synchrotron radiation. Dedication, enthusiasm and leadership are essential qualities.

\section{The Benefits}

The appointment will be at Principal Scientific Officer level on a salary scale from $£ 12,357$ to $£ 16,462$ per annum, depending on qualifications and experience. There is a non-contributory superannuation scheme, a generous leave allowance and a flexible working hours scheme.

If you want to know more, contact any of the people listed below. Application forms may be obtained from the Personnel Officer and returned quoting the reference DL/934 to:

The Personnel Officer, Daresbury Laboratory.

Science \& Engineering Research Council,

Daresbury, Warrington WA4 4AD.

Professor M. Hart: (925) 65000 Ext. 265 or

(61) 2734873 Ext. 14

Professor P.J. Duke: (925) 65000 Ext. 460

Personnel Officer: (925) 65000 Ext. 467

CLOSING DATE: 18 May 1986.

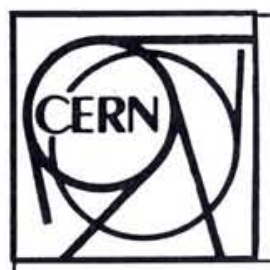

European Laboratory for Particle Physics

Laboratoire Européen pour la Physique des Particules

European Organization for Nuclear Research

Organisation Européenne pour la Recherche Nucléaire

CERN, the European Organization for Nuclear Research, located near Geneva, Switzerland, has a vacancy for a

\section{SENIOR SCIENTIFIC INFORMATION OFFICER}

Candidates should have a PhD in Physics or in a related field, a completed higher education in librarianship and experience in the management of a library or information centre. They should be familiar with modern library techniques. Very good knowledge of English and French is required.

The incumbent will take an active part in all questions related to scientific information, library services and scientific publications of CERN. He will maintain contact with relevant professional bodies and other libraries and information centres working in the same field.

The starting net salary, according to experience and qualifications, is approximately 7,500.- Swiss francs per month, plus family, non-resident and children's education allowances where applicable.

For application forms, please write to:

Head of Personnel, CERN, 1211 Geneva 23, Switzerland, quoting the reference TH-SIS. 
The most alarming property of Lplasmas is their scaling with heating power possibly in the form $\tau_{E} \sim P_{\mathrm{NI}}-0.5$. $\mathrm{H}$-plasmas of ASDEX do not show any degradation in confinement with increasing power until the high- $\beta$ stability limit is reached. $\beta$ is the ratio of thermal to magnetic energy and if this exceeds a figure of a few percent the plasma becomes grossly unstable. The limit in ASDEX seems to be the same for $L$ - and $\mathrm{H}$-plasmas.

The $\mathrm{H}$-mode is not an exceptional situation in $\mathrm{NI}$ heated discharges on ASDEX. It is produced over a wide operational range. There are two important limitations. The $\mathrm{H}$-mode requires a minimal heating power (1.2 MW) and the divertor configuration. In contrast if a limiter is used instead of the separatrix (which can alternatively be realised on ASDEX), only L-mode plasmas are found. None of the NI-heated limiter tokamaks in the world can operate in the

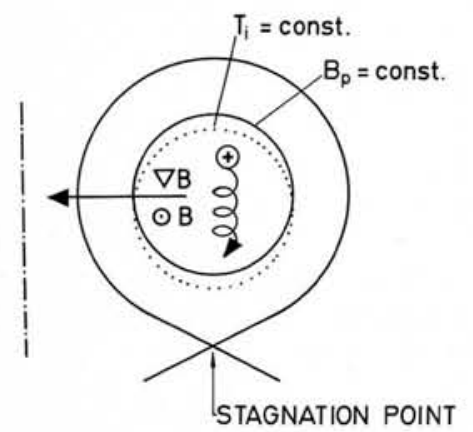

Fig. 3 - Poloidal plasma cross-section with toroidal field $B$, the field gradient $\nabla B$ and the orbit of a $\nabla B$-drifting ion. The displacement of an iso-T-surface (due to the ion drift) from a magnetic surface is schematically indicated.

$\mathrm{H}$-mode, while two American divertor tokamaks (DIII and PDX) were able to reproduce and confirm the ASDEX results.

\section{Physics of the $\mathrm{H}$-mode}

The existence of a lower power limit for the $\mathrm{H}$-mode and the preceding $\mathrm{L}$ phase indicates that the transition into the $\mathrm{H}$-phase is a threshold process which requires the plasma temperature to be increased beyond a certain level. The observation that the $\mathrm{H}$-mode cannot develop under contaminated plasma conditions when there is a high energy loss via radiation points toward the electron temperature as being the decisive parameter. Detailed studies with artificially introduced impurities indicate that the electron temperature at the plasma periphery triggers the $\mathrm{H}$-transition if it exceeds a threshold of $200-300 \mathrm{eV}$. Vice-versa, when the beam pulse is terminated, a second L-transition occurs in the phase of decreasing temperatures.
Fig. 4 - Operational diagram for $L$ and $H$ discharges. $z$ is the vertical plasma position; $z>0$ : upper $S N-, z=0: D N-$, and $z<$ $O$ : lower $\mathrm{SN}$-configuration. $P_{N 1}$ is the beam power into the vessel. The arrows indicate the ion grad- $B$ drift direction; the curve in $3 a$ denotes the operation boundary between $\mathrm{L}$-and $\mathrm{H}$-discharges and the insert in $3 b$ indicates the plasma configuration. Solid points: $\mathrm{H}$, circles: L-discharges.

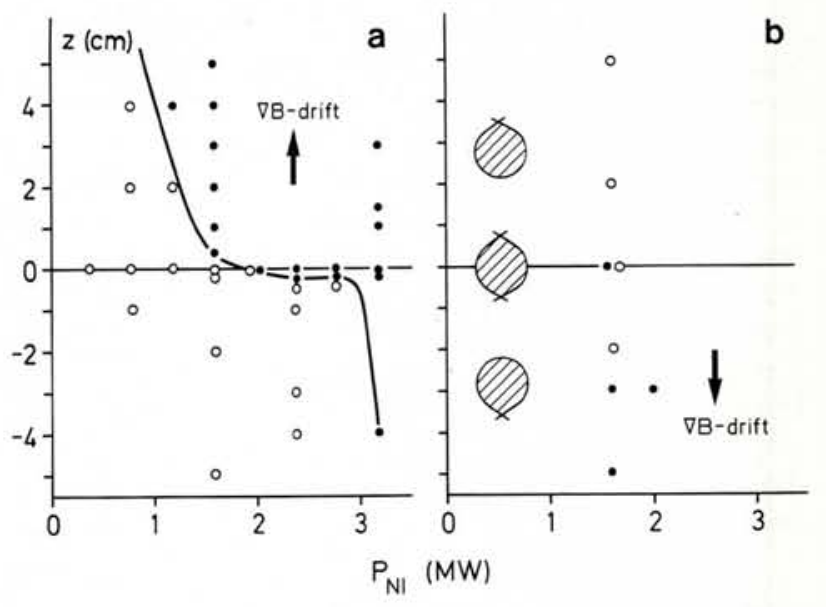

All measured parameters are different at the two transitions apart from the edge electron temperature. The most direct reference to the edge electron temperature comes from the observation that thermal pulses can trigger the $\mathrm{H}$-phase. Such pulses are continuously produced during a tokamak discharge in the plasma centre due to an instability localized at the $q=1$ magnetic surface which is distinguished by the property that magnetic field lines close after just one toroidal and poloidal circulation. Like the surface waves caused by a dripping faucet, the thermal pulses of so-called sawtooth relaxations emerge from the plasma centre, travel through the plasma, cross the separatrix and propagate along the scrape-off layer. Large sawtooth pulses can trigger the $\mathrm{H}$-phase on their arrival at the surface.

Because of the collisional coupling between electrons and ions the edge ion temperature also has to be high so that the electron temperature is able to exceed the threshold. Normally, the ion energy transport and therefore the ion temperature are determined at the edge by the atomic process of charge exchange between a cold atom from the outside and a hot ion at the periphery. The charge exchange process yields a cold ion to be heated up and a hot atom which may leave the plasma. It has been

found beneficial for the development of the mode to minimize these recycling processes.

Under conditions of low recycling, the ion heat flow is determined even at the separatrix by ion heat conduction and the absolute value of the ion temperature depends on the capricious movements of the ions and not on a simple atomic exchange process. In the inhomogeneous toroidal magnetic field, the gyromotion of the charged particles is not on closed circles because of a smaller gyroradius at the inner (high field) semi-circle giving rise to an additional vertical drift (see Fig. 3). Because of this grad-B drift movement, a vertical asymmetry is introduced where the magnetic surface no longer coincides with the iso- $T_{i}$ surface. This asymmetry does not play a role in the ion energy flow at the symmetric double-null configuration with two X-points. In the single-null configuration, however, the edge ion temperature is high if the ion grad-B drift is toward the $\mathrm{X}$-point and low in the opposite case. The ion thermal transport at the divertor boundary is analyzed in Ref. 5 .

In the experiment, a dependence of the $\mathrm{H}$-transition on the ion grad-B drift is indeed observed as summarized in the operational $z$ vs. $P_{\mathrm{NI}}$ diagram of Fig. $4 ; z$ is the vertical plasma position which determines the plasma configuration. In
Fig. 5 - Soft X-ray radiation during the L-phase with large saw teeth. Diode 1 views the scrapeoff layer, diode 2 mostly the plasma within the separatrix. One saw tooth gives rise to a transient H-phase.
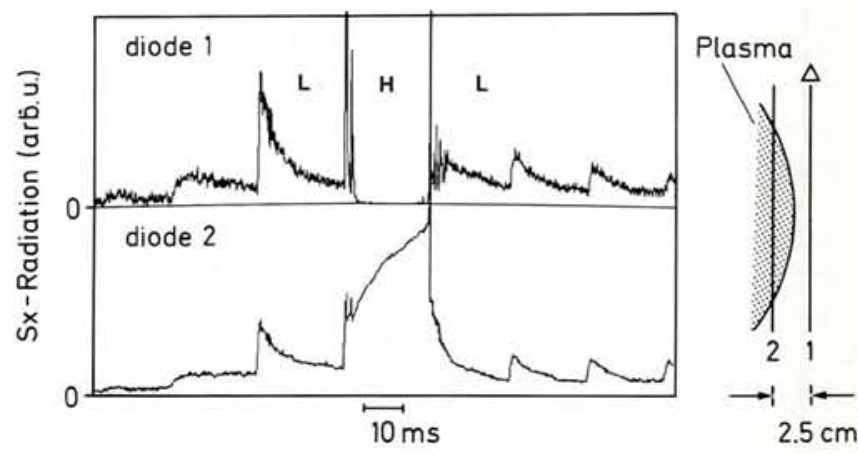
the centred position ( $z=0)$, both divertors are active yielding a double-null (DN) configuration with two X-points; for $z>0$ only the upper divertor is active, yielding an upper single null (SN) configuration with one $\mathrm{X}$-point (vice versa with $z<0$ ). Figure 4 shows that at high beam power, the $\mathrm{H}$-mode is obtained irrespective of the details of the configuration. In the upper SN-position $(z=4)$, the power limit of the $\mathrm{H}$-mode is 1.2 $\mathrm{MW}$; in the symmetric position, the effects of the ion grad-B drift just cancel, and the $\mathrm{H}$-mode power limit is 1.8 MW. In the lower SN-position, the ion grad-B drift is away from the X-point, giving rise to unfavourable edge conditions, and an $\mathrm{H}$-mode threshold close to $3 \mathrm{MW}$. When the toroidal field is turned around, the ion grad-B drift is downward. The $\mathrm{H}$-mode now develops in the opposite sense to the previous case. In summary, with the ion grad-B drift toward the $\mathrm{X}$-point, the single null position is superior to the double. This advantage is also seen in the length of the preceding L-phase which is typically shorter by a factor of two in the $\mathrm{SN}$ as compared with the DN configuration.

How do high electron and ion temperatures at the plasma edge give rise to improved confinement? This question has been studied by the technique described above, namely the transmission of heat pulses through the separatrix. Experimentally, a situation has been realised with a heating power below the power threshold but with large saw- tooth relaxations. Figure 5 shows how these repetitive thermal waves modulate the soft $X$-ray (SX) radiation along a chord just inside the separatrix and one just outside (The SX-signal depends primarily on electron temperature and density). A thermal pulse in the L-phase transiently enhances the SX-radiation. The behaviour is different in the case of a large saw tooth which triggers an $\mathrm{H}$ transition. Inside the separatrix, the thermal wave stagnates, leading to a temperature and density increase and in the scrape-off layer the SX-signal totally disappears: A transport barrier develops close to or at the separatrix which impedes the perpendicular particle and energy outflux $\left.{ }^{6}\right)$. The energy flux onto the divertor target plates is suddenly reduced to a low value comparable to the one during the preceding Ohmic phase, in spite of the fact that the power input into the discharge is 10 times as large as the Ohmic input. Therefore the plasma parameters in the divertor chamber drop to the Ohmic values. Steep pressure gradients develop at the plasma periphery causing pedestals in the $T_{\mathrm{e}}$ profile. The steep edge gradient gives rise to a new instability dubbed "edge localized mode" which has never been observed before. These instabilities transiently destroy the edge barrier, giving rise to large power and particle outfluxes, degrading the good $\mathrm{H}$-mode confinement somewhat but ultimately providing quasi-steady state conditions.

The actual reasons for the edge transport barrier are not yet known. A judgment of this deficiency, however, has to consider that 20 years of tokamak research have not yet unravelled the mysteries of electron heat transport. It could be that the peculiarities of the divertor magnetic field topology at the $X$-point (rapid spatial variation of field line length and inclination) stabilizes plasma turbulence. The study of the $\mathrm{H}$ mode might contribute to the understanding of tokamak transport.

In a technical sense, the divertor concept provides the expected solution to impurity control and offers with the thermal insulation layer at the edge a solution to confinement control. The electron heat diffusivity in the thermal barrier is about a factor of 50 lower than the usual value. Now we come back to the starting point of this article. The comparison with the Styrofoam layer may have been instructive but somewhat misleading. Even at degraded confinement, the perpendicular heat conductivity in a magnetically confined plasma is superior to that of Styrofoam.

\section{REFERENCES}

1. Lomas P., Europhys. News 16 (1985) 4. 2. Gibson P., Europhys. News 14 (1983) 4. 3. Keilhacker M., Europhys. News 11 (1980). 4. Wagner F., Becker G., Behringer K., Campbell D., Eberhagen A., et al., Phys. Rev. Lett. 49 (1982) 1408

5. Hinton F., to be published in Nucl. Fusion. 6. Wagner F., Fußmann G., Grave T., Keilhacker M., Kornherr M., Phys. Rev. Lett. 53 (1984) 1453.

\section{ICTP Donation Programme}

During the last 20 years the International Centre for Theoretical Physics, through its various scientific programmes has tried so sustain and promote research activities in different scientific disciplines in developing countries whose needs are very great. Scientists there suffer from lack of library and laboratory facilities. Because of this, in recent years the Centre has made attempts to provide them with books, journals and equipment through the programmes described below:

\section{Book Donation Programme:}

Several appeals have been made to libraries, publishing companies and individuals, asking them to donate any books, journals and proceedings they no longer needed, with the International Centre acting as broker. The response has been very encouraging as the Centre is distributing every year approximately 14000 journals, 4000 procee- dings and 2000 scientific books to more than 200 Institutes in 80 developing countries. The value offered to the Centre by different donors can roughly be estimated in the range of 600000 US dollars every year.

\section{Equipment Donation Programme}

This scheme was initiated in 1984. An appeal was made by Professor Abdus Salam to the leading laboratories in Europe for the donation of surplus equipment. This has had positive responses and the physics departments in the universities of Dacca Bangladesh, Rajshahi Bangladesh, Peshawar Pakistan, Khartoum Sudan, Ghana Ghana have received in the range of 50 to 100 different items of equipment which have been donated by the Rutherford Appleton Laboratory in the UK and the Seibersdorf Laboratory in Austria. The Nuclear Research Centre in Jülich, FRG, donated a Microfiche Reader Printer which was sent directly to the Physics Department, University of Ilorin, Nigeria.

In 1985, CERN also agreed to join this programme and has offered a considerable amount of equipment which is now being shipped to institutes in Argentina, Brazil, Malaysia and Pakistan. These institutes will then distribute the equipment among other institutes in their country.

Although the Centre is making every effort to try and ameliorate the drastic situation in the universities in developing countries, still more can be done. Nowadays attempts are being made in many developing countries to establish science as a viable discipline of study. These countries need to be helped and it is therefore unnecessary to stress how useful this donation programme is and how precious your help can be. Those interested in helping us could kindly notify

\section{H.R. Dalafi}

International Centre for Theoretical Physics P. O. Box 586, I-34100 Trieste 